\title{
PAPER
}

\section{Plasma generation and processing of interstellar carbonaceous dust} analogs

To cite this article: R J Peláez et al 2018 Plasma Sources Sci. Technol. 27035007

View the article online for updates and enhancements. 


\title{
Plasma generation and processing of interstellar carbonaceous dust analogs
}

\author{
R J Peláez ${ }^{1}$, B Maté ${ }^{1}$, I Tanarro ${ }^{1}$, G Molpeceres ${ }^{1}$, M Jiménez-Redondo ${ }^{2}$, \\ V Timón ${ }^{1}, \mathbf{R}$ Escribano ${ }^{1}$ (1) and V $\mathbf{J}$ Herrero $^{1}$ (1) \\ ${ }^{1}$ Instituto de Estructura de la Materia (IEM-CSIC), Serrano 121-123, E-28006, Madrid, Spain \\ ${ }^{2}$ Centro de Física da Universidade do Minho, Universidade do Minho, 4710-057, Braga, Portugal \\ E-mail: v.herrero@csic.es
}

Received 31 October 2017, revised 24 December 2017

Accepted for publication 22 February 2018

Published 14 March 2018

\begin{abstract}
Interstellar (IS) dust analogs, based on amorphous hydrogenated carbon (a-C:H) were generated by plasma deposition in radio frequency discharges of $\mathrm{CH}_{4}+\mathrm{He}$ mixtures. The a-C:H samples were characterized by means of secondary electron microscopy, infrared (IR) spectroscopy and UV-visible reflectivity. DFT calculations of structure and IR spectra were also carried out. From the experimental data, atomic compositions were estimated. Both IR and reflectivity measurements led to similar high proportions $(\approx 50 \%)$ of $\mathrm{H}$ atoms, but there was a significant discrepancy in the $\mathrm{sp}^{2} / \mathrm{sp}^{3}$ hybridization ratios of $\mathrm{C}$ atoms $\left(\mathrm{sp}^{2} / \mathrm{sp}^{3}=1.5\right.$ from IR and 0.25 from reflectivity). Energetic processing of the samples with $5 \mathrm{keV}$ electrons led to a decay of IR aliphatic bands and to a growth of aromatic bands, which is consistent with a dehydrogenation and graphitization of the samples. The decay of the $\mathrm{CH}$ aliphatic stretching band at $3.4 \mu \mathrm{m}$ upon electron irradiation is relatively slow. Estimates based on the absorbed energy and on models of cosmic ray (CR) flux indicate that CR bombardment is not enough to justify the observed disappearance of this band in dense IS clouds.
\end{abstract}

Keywords: plasma deposition, amorphous hydrogenated carbon, electrron bombardment, interstellar dust analogs

\section{Introduction}

Interstellar (IS) dust is formed in the envelopes of asymptotic giant branch stars and in supernova explosions [1]. It is largely made of grains of silicates and of carbonaceous materials with variable sizes and compositions [2]. Most information on the nature of IS dust is derived from infrared (IR) absorption spectra. Early observations of an absorption band at $3.4 \mu \mathrm{m}$ $\left(\approx 2950 \mathrm{~cm}^{-1}\right)$ [3] and the subsequent comparison of this band with laboratory data suggested that hydrogenated amorphous carbon was a major component of dust in the diffuse interstellar medium (DIM) [4]. In contrast, in dense molecular clouds, the $3.4 \mu \mathrm{m}$ band is not observed.

Hydrogenated amorphous carbon, usually abbreviated as a-C:H or HAC, refers to a group of carbonaceous solids with different proportions of $\mathrm{sp}^{2}$ and $\mathrm{sp}^{3}$ hybridized carbon atoms $\left(\mathrm{Csp}^{2}, \mathrm{Csp}^{3}\right)$, and different $\mathrm{C} / \mathrm{H}$ ratios. The properties of a-C: $\mathrm{H}$ can vary between those of diamond, graphite and polymeric hydrocarbons, and have deserved the attention of materials' physicists over the last few decades [5-8]. The actual nature of the a-C:H material in the dust grains of the DIM is not known with precision. Two competing models have been advanced. The first of these models assumes a structure formed by small aromatic islands linked by aliphatic chains, with possible olefinic contributions [9-11]. The other model assumes a larger graphitic structure, with small aliphatic contributions $[12,13]$. The abundant $3.4 \mu \mathrm{m}$ band is mostly assigned to $\mathrm{CH}$ stretching vibrations of $\mathrm{CH}_{2}$ and $\mathrm{CH}_{3}$ aliphatic groups and is related to weaker absorptions at 6.8 and $7.3 \mu \mathrm{m}$ corresponding to bending motions of the same functional groups which have also been observed in some cases. Given the uncertainty about the structure of the possible carbon carriers, estimates of the amount of elemental carbon contained in IS dust range from 5\% to $30 \%$ [9].

Analogs of IS carbonaceous grains have been built in the laboratory using various techniques and precursors [14], including laser ablation of graphite, laser pyrolysis of a precursor gas [15], UV irradiation of condensed hydrocarbons 
[9] or plasma enhanced chemical vapor deposition (PECVD) of gas phase hydrocarbons $[16,17]$. The latter technique has proven very useful to yield a-C:H samples with IR spectra giving a good match to astronomical observations. Whereas the mechanisms of formation of a-C:H in the laboratory are certainly different from those of dust formation in the IS medium, it is expected that solids with a similar structure can be formed in both cases. In space, it is generally assumed that dust cores are formed in the ejecta of evolved stars and are then modified as they travel through the IS medium and interact with UV photons, cosmic rays and hydrogen atoms, but the detailed mechanisms are not known. Astronomical models assume that these processes give rise to solids with mixed aromatic and aliphatic structures, but as commented on in the previous paragraph there is no unanimity on the aliphatic/aromatic proportion or even in the preferential location (core or mantle) of aliphatic functional groups [12, 18]. Laboratory work is needed to help clarify these issues. In the laboratory, different sorts of a-C:H films can be formed through PECVD by varying the deposition conditions. In a previous work by our group [19], we discussed the different properties of two types of films deposited with different atom and radical flows and substrate temperatures. Here, we have selected the deposition conditions that led to the best IS dust analog.

The formation and destruction of the aliphatic structures responsible for the IS $3.4 \mu \mathrm{m}$ band have been attributed to the interaction of carbonaceous solids with hydrogen atoms, UV photons and cosmic rays (CRs) [2, 12]. Laboratory studies have shown that UV photons and highly energetic particles (analogs of CRs) lead to dehydrogenation and graphitization of dust analogs [20-24], whereas interaction with $\mathrm{H}$ atoms reconstructs aliphatic structures [25]. In the DIM, the effects of the intense UV field prevail over those of CRs, but in denser regions, shielded from UV radiation, cosmic rays can become relevant. In this respect, experimental works by Mennella et al [21] and by Godard et al [22] led to different conclusions. The former group left open the possibility of a significant role of CRs for the destruction of the carriers of the $3.4 \mu \mathrm{m}$ band, whereas the latter concluded that CRs were not relevant.

In previous studies by our group [19, 26], we investigated the aliphatic/aromatic content of the predominant dust carriers of carbonaceous IS dust and the effect of CRs on the carriers of the $3.4 \mu \mathrm{m}$ band in dense clouds. Our results, based on a comparison with theoretical calculations [19] and on electron irradiation experiments [26] favored a predominant dust structure with a significant aliphatic content and corroborated the results of Godard et al [22] on the irrelevance of CRs for the disappearance of the $3.4 \mu \mathrm{m}$ band carriers in dense clouds.

In this work, we extend our previous investigation on the characterization and processing of IS dust analogs generated by glow discharges of $\mathrm{CH}_{4}$ containing mixtures. IR spectroscopy, secondary electron microscopy (SEM) and UV-visible reflection measurements are used to analyze the a-C:H deposits produced by PECVD. High energy electron bombardment of the deposited samples is used to simulate the effect of cosmic rays. The changes in the deposits induced by electron processing are studied using the above mentioned techniques, and astrophysical implications are reviewed.

\section{Experimental}

In an inductively coupled radio frequency (RF) discharge, films of a-C:H of variable thickness between 380-1540 nm were deposited on IR transparent, low resistivity, Si substrates of $2.54 \mathrm{~cm}$ diameter and $1 \mathrm{~mm}$ thickness. The discharge reactor consists of a Pyrex tube $(30 \mathrm{~cm}$ length, $4 \mathrm{~cm}$ diameter) with a 10 turns $\mathrm{Cu}$ coil, placed externally around the central part of the tube and fed by a $13.56 \mathrm{MHz} \mathrm{RF}$ generator (Hüttinger PFG $300 \mathrm{RF}+$ matchbox PFM $1500 \mathrm{~A}$ ), which was kept at a constant power of $40 \mathrm{~W}$. The reactor was evacuated at one of the ends by a rotary vacuum pump with a regulating valve down to background pressures of $5 \times 10^{-3}$ mbar. The gas input was supplied at the other end. Mixtures of $\mathrm{CH}_{4}(5 \mathrm{sccm})$ and $\mathrm{He}(10 \mathrm{sccm})$ were introduced into the reactor up to a pressure of 0.3 mbar, measured by an absolute capacitance manometer. Typical residence times were $\approx 3 \mathrm{~s}$. These deposition conditions had proven adequate to generate hydrogen-rich a-C:H samples with a significant aliphatic content, whose IR spectra were in good agreement with astronomical observations [19]. Under these conditions, $\mathrm{CH}_{4}$ dissociated in the plasma up to $\approx 90 \%$, as determined by the mass spectra obtained with a differentially pumped quadrupole mass spectrometer (Hiden HAL $201 \mathrm{RGA})$. The Si substrates were supported on thin glass plates to prevent film deposition on the lower face. The light intensity of a He-Ne laser reflected on the center of the samples and detected by a photodiode allowed to follow the periodic interference signal of the growing films and to make an in situ first rough estimate of their thicknesses, which increased linearly with time. Depending on the final thickness, deposition times varied between 15 and $60 \mathrm{~min}$.

Normal incidence IR transmission spectra of the deposited samples were then recorded ex situ with a Bruker Vertex70 FTIR spectrometer. The spectra were co-added from 300 runs at a nominal resolution of $4 \mathrm{~cm}^{-1}$. For the used plasma deposition conditions, the band shape of the IR spectra of the deposits was very repetitive and the integrated absorbance was proportional to the deposition time. IR interference fringes could be used to estimate the width of samples thicker than $\approx 800 \mathrm{~nm}$. An uncertainty of $\pm 30 \mathrm{~nm}$ is estimated in these values. The geometrical width distributions of the films along their surfaces were also determined with an optical microscope used in quasi-normal incidence, coupled to a small visible spectrometer (Ocean QE65000). The oscillations in the visible spectra were employed even for the characterization of the thinner samples, with thickness uncertainties of $\pm 10 \mathrm{~nm}$. For these estimations, both from the visible and IR measurements, the refractive index was assumed to be $n=1.7$ (see below). The thickness was found to be homogeneous over the central part of the disk and then decayed 

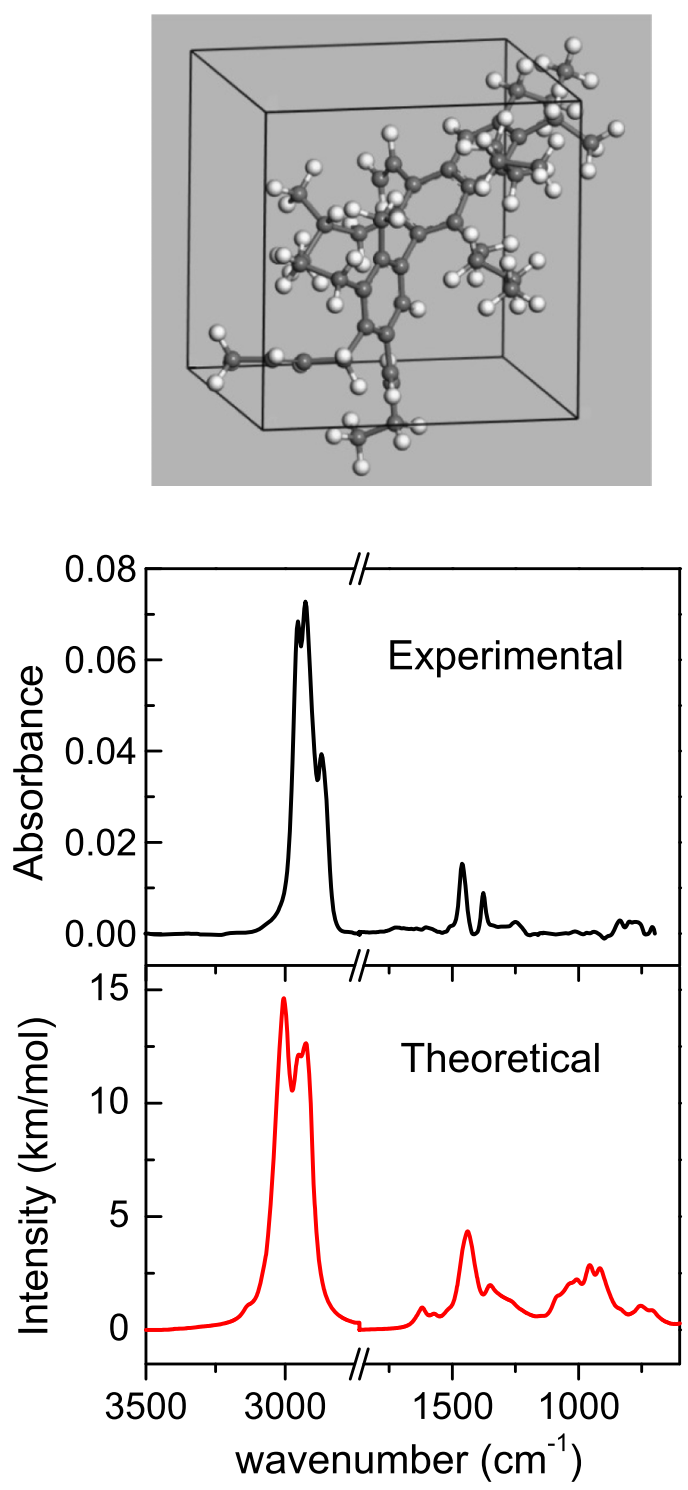

Figure 1. Upper panel: approximate a-C:H structure for the predominant component of interstellar dust based on the model suggested by Dartois et al [9]. Lower panel: comparison of the theoretical IR spectrum for the structure in the upper panel with that of a typical a-C:H sample (thickness $\approx 570 \mathrm{~nm}$ ) deposited in the present work.

smoothly toward the edges (typically, down to $\sim 20 \%-30 \%$ of the central thickness).

SEM microscopy was also used for the analysis of the samples. Some of the deposited substrates were fractured in order for SEM cross section images to be taken, including the $\mathrm{Si}$ substrate and a-C:H film. From these images the film thickness could be measured too with an accuracy of $\pm 10 \mathrm{~nm}$. The thickness values measured with the SEM images were in good agreement with the estimates from the $\mathrm{He}-\mathrm{Ne}$ laser, and the visible and IR spectra.

The substrates were weighted with a high-precision microbalance before and after film deposition. From the increase in weight and the thickness profile of the films, a density of $1.1 \pm 0.1 \mathrm{~g} \mathrm{~cm}^{-3}$ was estimated for our a-C:H samples.
Visible and UV reflectivity spectra of films were taken with the reflectance accessory of a CARY 5000 spectrophotometer with an integrating sphere. The samples were illuminated at $8^{\circ}$ off-normal incidence. Most of the reflected light $(>95 \%)$ comes from the specular component. The diffuse component has a low signal to noise ratio and is neglected in the analysis. From now on, we will refer to the specular reflectivity as the reflectivity of the sample.

For the study of energetic processing, the a-C:H samples were transferred to a high-vacuum chamber (see [17] for details of the set-up). The samples were placed in an appropriate $\mathrm{Cu}$ holder mounted in a rotatable flange. The viewing window of the $\mathrm{Cu}$ holder restricted the area for processing and observation to the central part of the sample, an inner circle of $1 \mathrm{~cm}$ diameter. During processing, the substrates were made to face alternatively the IR beam from a FTIR spectrometer or the electron beam from an electron gun inside the chamber. The samples were irradiated with a $7.9 \times 10^{12}$ electron $\mathrm{cm}^{-2} \mathrm{~s}^{-1}$ homogeneous flux of $5 \mathrm{keV}$ electrons at normal incidence. At given time intervals, electron irradiation was interrupted and the sample was rotated to record IR transmission spectra at normal incidence. The chamber pressure during processing was $\approx 1 \times 10^{-7}$ mbar. All the experiments were carried out at room temperature.

\section{Results and discussion}

\subsection{Theoretical simulations}

As in previous investigations, we have developed different models to simulate a-C:H samples, working at density functional theory (DFT) level of theory as implemented in the CASTEP code $[27,28]$. The electronic structure of the proposed analogs is determined within the Born-Oppenheimer approximation and this allows the calculation of a whole set of properties including molecular structure and spectra. As mentioned in the introduction, the published literature considers basically two types of models, with a different proportion of aromatic and aliphatic structures [9, 13]. The relative extent of these structures, together with the $\mathrm{H}$ content, and its density, are the key parameters to describe the nature of the sample. Theoretical models allow studying many possibilities covering a range of values of these magnitudes.

The quality of the models can be tested by comparing the predicted IR spectra with the laboratory observations. The two main spectral regions are that covering the $\mathrm{C}-\mathrm{H}$ stretching modes, around $2950 \mathrm{~cm}^{-1}$, and those where $\mathrm{C}-\mathrm{C}$ and $\mathrm{C}=\mathrm{C}$ stretchings, plus $\mathrm{CH}_{n}$ bendings appear, between 1000 and $1600 \mathrm{~cm}^{-1}$. Thus, the comparison between observation and prediction is not always easy, as different models yield predictions that sometimes reproduce better one spectral region and worse the other one. For the a-C:H samples produced in the present work, we have found the best agreement for structures based on that of Dartois et al [9], consisting in a number of small aromatic rings linked by short aliphatic chains, with relative content of $\mathrm{Csp}^{3}, \mathrm{Csp}^{2}$ and $\mathrm{H}$ atoms of $0.23,0.20$ and 0.57 , respectively. Reasonably good 

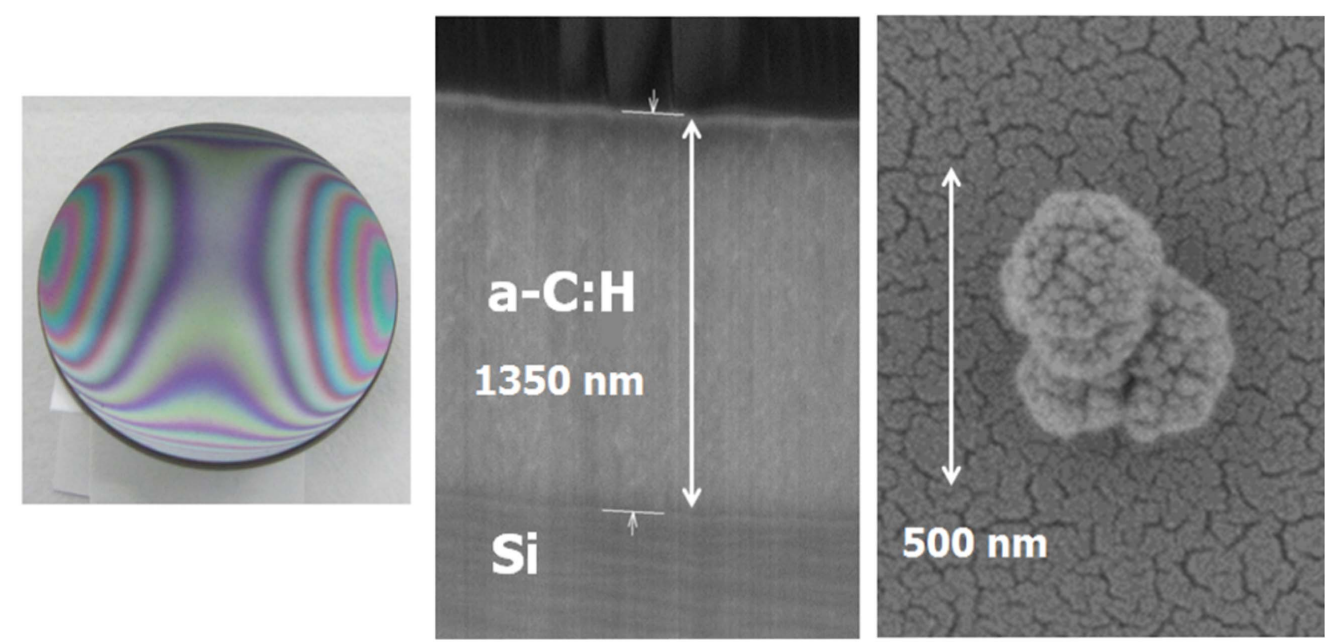

Figure 2. Left panel: photograph of an a-C:H sample deposited on a Si substrate. Central panel: SEM image of the cross section of a deposited a-C:H film. Right panel: SEM image (planar view) of dust particles generated in the gas-phase of the plasma and collected on the sample.

reproducibility of the experimental spectra is achieved for structures with densities between 0.7 and $1.0 \mathrm{~g} \mathrm{~cm}^{-3}$, which are slightly smaller than that estimated for experimental a-C: $\mathrm{H}$ samples, $1.1 \pm 0.1 \mathrm{~g} \mathrm{~cm}^{-3}$. The theoretical structure of density $0.7 \mathrm{~g} \mathrm{~cm}^{-3}$ is also calculated with the highest stability, in terms of the total energy of the system, whereas that energy increases by $0.60 \mathrm{eV}$ for the sample of density $1.0 \mathrm{~g} \mathrm{~cm}^{-3}$.

We reproduce in figure 1 a schematic representation of the structure that we have developed based on the Dartois et al model [9], in the upper panel, and a comparison of the corresponding predicted spectrum with one recorded in our laboratory for a typical a-C:H sample, in the lower one.

\subsection{Plasma deposition and morphology}

Polymerization leading to a-C:H formation in the laboratory plasmas takes place both at the exposed surfaces and in the gas-phase. In the first case, thin films are formed, and in the latter case, dust particles. Typical deposits are shown in figure 2. The decrease in the film thickness toward the edges of the substrate mentioned above gives rise to the interference fringes visible in the left panel. At the center of the samples, the films are highly homogenous in depth and have flat surfaces, as shown in the middle panel of the figure. This crosssection SEM micrograph, taken from a broken cleaved sample, allows also the measurement of the film thickness. A SEM planar view of an Au covered sample with dust particles is shown in the right panel. They have typically cauliflower shapes with diameters between 100 and $500 \mathrm{~nm}$ and are similar to the particles generated in dusty plasmas with hydrocarbon precursors [16]. Under our experimental conditions, the amount of dust particles formed is small and we will not consider them further in this work.

The effects of electron bombardment on the samples are shown in figure 3 . In the left panel of the figure, the irradiated area, which is defined by the window of the sample holder, is clearly appreciable. It corresponds to the central and most homogenous part of the film. The cross section SEM view of an irradiated film and a simulation of the tracks of the bombarding electrons are shown in the middle and right panels, respectively. The penetration depth of the $5 \mathrm{keV}$ electrons in the a-C:H samples was simulated using the Monte Carlo simulations of electron trajectories in solids (CASINO) code $[29,30]$. For the simulations, we have taken the measured density $\left(1.1 \mathrm{~g} \mathrm{~cm}^{-3}\right)$ and have assumed an $\mathrm{H} / \mathrm{C}$ ratio of 1 , in analogy with that of Godard et al [22], who used also RF discharges of methane for the generation of their samples. These values are also consistent with the results of other authors for similar methane plasmas [31]. Samples of a-C:H with higher densities and lower hydrogen proportions can be formed using other deposition procedures $[7,8,32]$. They have higher densities than that of the present films and offer more resistance to electron penetration. For a density of $1.5 \mathrm{~g} \mathrm{~cm}^{-3}$ and $(\mathrm{H} / \mathrm{C})=0.3$, the penetration depth estimated with CASINO is roughly $260 \mathrm{~nm}$. The analysis of IR and reflectivity measurements in this work (see below) corroborate the approximate $(\mathrm{H} / \mathrm{C})=1$ proportion assumed for our films.

As shown in the upper part of the central panel, electron bombardment changes the morphology of the irradiated material. The electron penetration depth observed in the SEM image is approximately $750 \mathrm{~nm}$ for our experimental conditions. The penetration depth estimated with CASINO is somewhat $(\approx 15 \%)$ smaller, but still gives a good approximation to the observations.

\subsection{IR absorption measurements}

The changes in sample composition associated with the morphological changes just shown can be observed in the evolution of the IR spectra displayed in figure 4 . They correspond to an irradiated a-C:H layer with a thickness of $570 \mathrm{~nm}$, well below the electron penetration depth.

Three spectral regions are shown in this figure: (left) the region of the $\mathrm{CH}$ stretching vibrations around $2950 \mathrm{~cm}^{-1}$ (i.e. the $3.4 \mu \mathrm{m}$ band of astronomical observations), (middle) the region between 1700 and $1300 \mathrm{~cm}^{-1}$, which contains the 

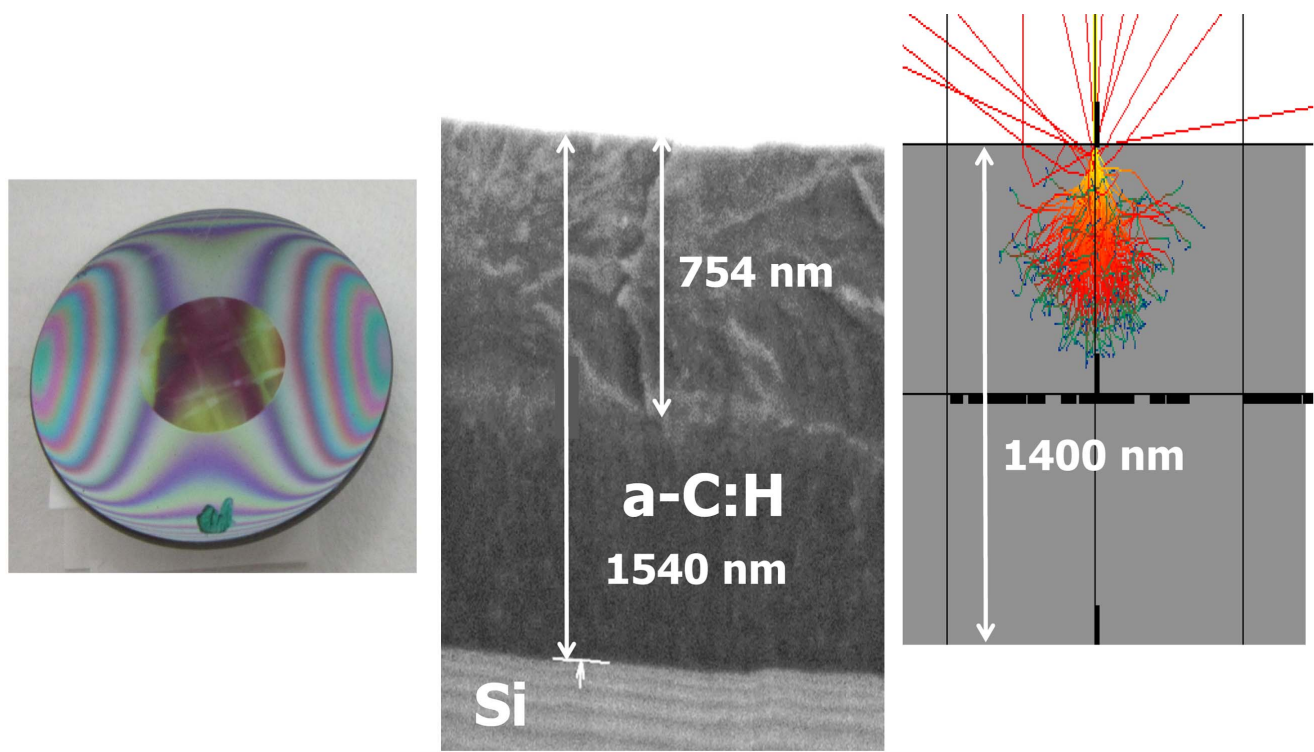

Figure 3. Left panel: photograph of an a-C:H sample after irradiation of its central part by $5 \mathrm{keV}$ electrons (fluence $4.3 \times 10^{17} \mathrm{e} \mathrm{cm}^{-2}$ ). Middle panel: SEM image of the cross section of an irradiated film. Right panel: CASINO simulation of the penetration of $5 \mathrm{keV}$ electrons in the a-C:H region.

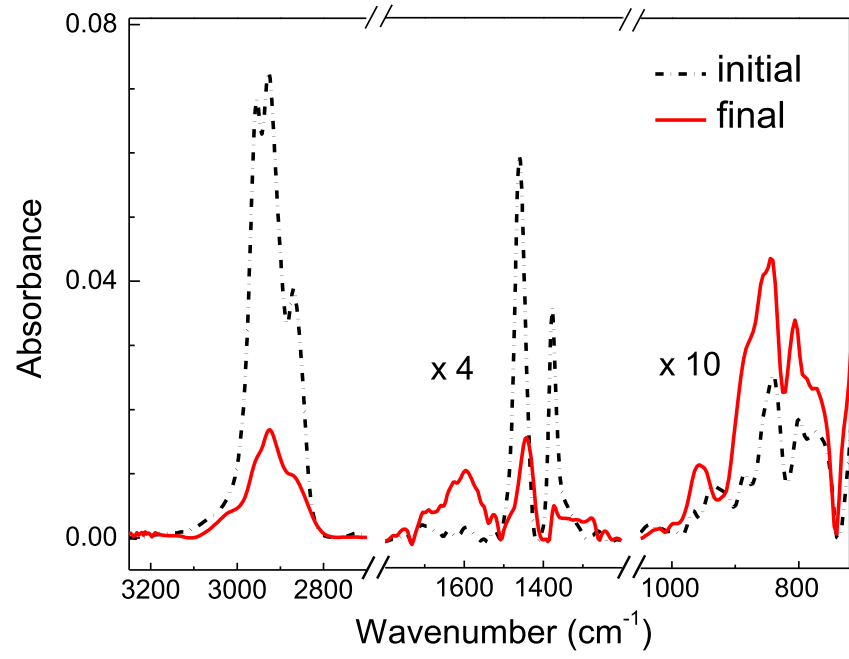

Figure 4. Black dashed curve: IR spectrum of a-C:H before electron irradiation. Red curve: spectrum after electron irradiation (fluence $2 \times 10^{17}$ electrons $\mathrm{cm}^{-2}$ ). The irradiated layer was $\approx 570 \mathrm{~nm}$ thick.

$\mathrm{C}=\mathrm{C}$ stretch vibrations of aromatic rings, as well as the bending motions of $\mathrm{CH}_{3}$ and $\mathrm{CH}_{2}$ aliphatic groups, and (right) the $1000-700 \mathrm{~cm}^{-1}$ interval with the $\mathrm{CH}$ in- and out-of-plane aromatic bendings [33]. In a previous work by our group [26], we investigated the decrease of the aliphatic features and, in particular, of the intense aliphatic $\mathrm{CH}$ stretch band at $2950 \mathrm{~cm}^{-1}$ upon electron impact, but the signal to noise ratio was not good enough to draw firm conclusions on the evolution of the aromatic bands. At present, the instrumental noise has been appreciably reduced with respect to that of previous experiments with a more stable and effective dry air purge. With our improved experimental set-up, the neat growth of the aromatic bands at $\approx 1600 \mathrm{~cm}^{-1}$ and in the region between 1000 and $700 \mathrm{~cm}^{-1}$ is made evident. The evolution of the

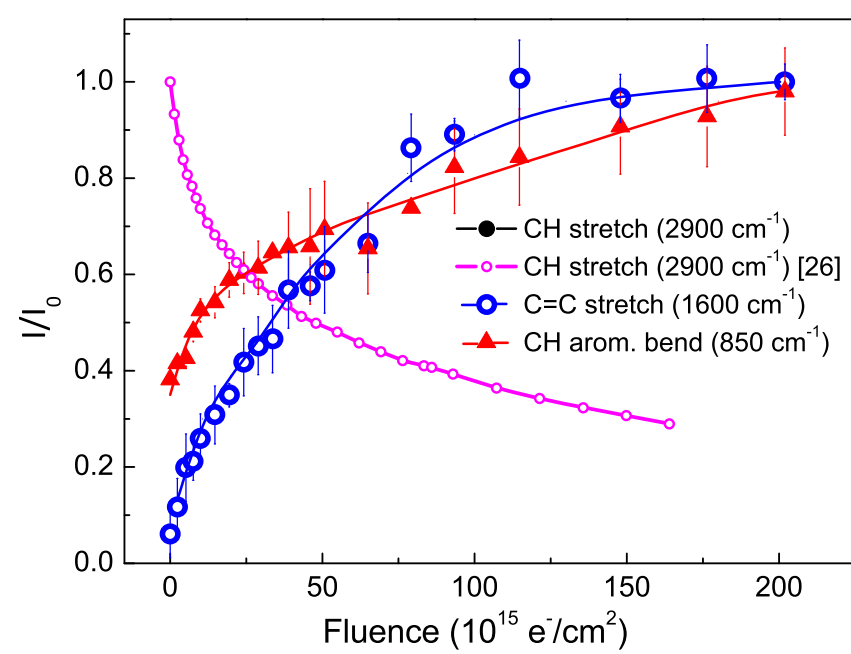

Figure 5. Evolution of the integrated intensity of characteristic a-C:H bands upon electron irradiation. Black dots: $\mathrm{CH}$ stretching band (3100-2750 $\mathrm{cm}^{-1}$ ); small pink circles: $\mathrm{CH}$ stretching band from [26]; blue circles: $\mathrm{C}=\mathrm{C}$ aromatic stretching $\left(1670-1500 \mathrm{~cm}^{-1}\right)$; red triangles: aromatic $\mathrm{CH}$ bending vibrations $\left(950-750 \mathrm{~cm}^{-1}\right)$. The integrated intensities are normalized to 1 at the maximum. The lines are just to guide the eye.

intensity of three characteristic bands with electron bombardment is shown in figure 5.

The intensity of the $\mathrm{CH}$ stretching band shows a monotonic decrease with electron fluence (black closed circles). It is in very good agreement with our previous measurements [26] (small pink circles), which are also included in the figure. As discussed in that work, the decrease in the intensity of the $\mathrm{CH}$ stretching band can be successfully accounted for by a dehydrogenation model [34, 35] originally developed to explain the hydrogen release observed upon ion irradiation of $\mathrm{a}-\mathrm{C}: \mathrm{H}$. The model assumes that hydrogen leaves a-C:H in molecular form. When a $\mathrm{CH}$ bond is broken, the hydrogen 
Table 1. Atomic composition of plasma generated a-C:H before and after energetic processing with $5 \mathrm{keV}$ electrons (fluence $\approx 2.1 \times 10^{17}$ $\mathrm{e} \mathrm{cm}^{-2}$ ). The compositions were derived from the band analysis of IR spectra (see text and [19]). The values listed correspond to the average of measurements performed on two fully irradiated layers of different thickness (380 and $570 \mathrm{~nm}$, respectively).

\begin{tabular}{lcc}
\hline & \multicolumn{2}{c}{ Composition from IR band profile decomposition } \\
\cline { 2 - 3 } & $\begin{array}{c}\text { a-C:H as deposited by } \\
\text { PECVD }\end{array}$ & $\begin{array}{c}\text { a-C:H after electron } \\
\text { bombardment }\end{array}$ \\
\hline $\mathrm{C} \mathrm{sp}^{3}$ & 0.18 & 0.11 \\
$\mathrm{C} \mathrm{sp}^{2}$ & 0.27 & 0.45 \\
$\mathrm{H}$ & 0.55 & 0.44 \\
\hline
\end{tabular}

atom diffuses within the solid, where it can recombine with another $\mathrm{H}$ atom to produce $\mathrm{H}_{2}$, or be trapped by a reactive site in the bulk material. Once formed, the $\mathrm{H}_{2}$ molecules are stable and diffuse out of the solid. Under continued irradiation, the $\mathrm{H}$ atom density drops to a point where recombination stops, when $\mathrm{H}$ atoms from broken $\mathrm{CH}$ bonds react at a solid active site before finding another $\mathrm{H}$ atom. The decrease of the aliphatic $\mathrm{CH}$ stretch band with increasing electron fluence is accompanied by a concomitant growth of the aromatic $\mathrm{CC}$ and $\mathrm{CH}$ bending bands. The increase in the intensity of these bands indicates that the electron bombardment not only destroys aliphatic groups through hydrogen depletion, but also induces the rearrangement of the carbon network into aromatic structures. The growth of the aromatic $\mathrm{CH}$ bending band $\left(\approx 950-750 \mathrm{~cm}^{-1}\right)$ indicates also that some of the $\mathrm{H}$ atoms from the break-up of aliphatic $\mathrm{CH}$ bonds end up reacting with $\mathrm{C}$ atoms of the newly created aromatic units. The creation of aromatic $\mathrm{CH}$ bonds is also corroborated by the appearance of a bump at the high wavenumber edge of the $2950 \mathrm{~cm}^{-1}$ band of the processed sample, which is attributable to the aromatic $\mathrm{CH}$ stretching vibration located at about $3050 \mathrm{~cm}^{-1}$.

The possibility of extracting structural information on a-C:H samples from IR spectra has been recently considered in the astrophysical community [10, 12], where IR spectra constitute the main observational data on IS. To determine the relative content of $\mathrm{Csp}^{3}, \mathrm{Csp}^{2}$ and $\mathrm{H}$ atoms in a-C:H samples, the $\mathrm{CH}$ stretching band at $\approx 2950 \mathrm{~cm}^{-1}$ can be decomposed into various sub-bands associated with the aliphatic $\mathrm{CH}_{2}$ and $\mathrm{CH}_{3}$ groups and with aromatic $\mathrm{CH}$. In addition, the $\mathrm{C}=\mathrm{C}$ aromatic band at $1600 \mathrm{~cm}^{-1}$ can be used to estimate the contribution of polyaromatic networks to the global structure. Literature band strengths from known aliphatic and aromatic molecules can then be used for the evaluation of the sample composition. We have applied this band decomposition procedure to the a-C:H IR spectra using the band strengths values given by Chiar et al [12] (see [19] for details of the method) and have derived the values listed in table 1, which reflect the dehydrogenation and growth of aromatic structures upon electron bombardment observed in the experiment.

The composition of the unprocessed a-C:H is in reasonably good agreement with that of our previous work for similar deposition conditions [19] and with the theoretical calculations commented on above (see discussion of figure 1). However, the lack of precise band strengths and some ambiguity in the sub-bands assumed in the band profile decomposition for this type of materials limit the accuracy of the analysis based on IR spectra. In fact, a recent comparison with theoretical calculations by our group [19] suggests that the values derived with this method might be biased toward graphitic structures, due possibly to the fact that the literature values overestimate the band strength of the $\mathrm{C}=\mathrm{C}$ stretching in $\mathrm{a}-\mathrm{C}: \mathrm{H}$.

\subsection{UV-visible reflectivity measurements}

In an attempt to shed more light on this point, we have also estimated the atomic content of our samples using data from UV-visible reflectivity measurements. For these experiments we have used five samples with a-C:H layer thickness values between $\approx 380$ and $1540 \mathrm{~nm}$. As an illustration, we display in figure 6(a) the reflectivity spectrum of a non-irradiated a-C:H layer of $750 \mathrm{~nm}$. The oscillations of the signal are due to interferences and indicate that the film is transparent in the visible region and that its thickness is comparable to the wavelength. The reflectivity spectrum was fitted by an optical multilayer model using WVASE software (WVASE32TM, J. A. Woollam Co. Inc.) to determine the optical indices. In this optical model the Si substrate, which is about $1 \mathrm{~mm}$ thick, can be treated as infinite. Therefore, this non-irradiated material can be described with a three-layer structure: air/non processed a-C:H/Si.

The optical properties of a-C:H are described by the combination of Cauchy and Tauc-Lorentz models. The first of these models describes the optical indices of dielectrics and the second is designed for the modeling of amorphous materials, whose absorption is practically zero below the optical band gap energy (Tauc gap), $E_{\mathrm{g}}$. Further details about the models can be found in [36]. The simulated reflectivity spectrum, (red curve in figure 6(a)) is in very good agreement with the experimental one. The corresponding optical indices are shown in figure $6(\mathrm{c})$ as continuous lines. The refractive index, $n$, follows approximately Cauchy's dispersion law. It takes values $n=1.85$ for $E=6 \mathrm{eV}$ and $n=1.69$ for $E=1.5 \mathrm{eV}$. The extinction coefficient, $k$, reaches a maximum value of 0.074 for $E=5.3 \mathrm{eV}$, and becomes zero at the optical band gap energy $\left(E_{\mathrm{g}}=2.7 \mathrm{eV}\right)$. For photon energies below this value, i.e. for photon wavelengths larger than $452 \mathrm{~nm}$, which includes most of the visible, the material is transparent.

The reflectivity spectrum of an irradiated sample of $1540 \mathrm{~nm}$ is shown in figure 6(b). It corresponds to the sample shown in the middle panel of figure 3. A comparison of figures 6(a) and (b) shows that the spectrum of the processed sample has a much higher absorption in the visible. In this case, the penetration depth of the electrons is lower than the layer thickness, and thus an interlayer of processed and nonprocessed material must be considered. To derive the simulated spectrum of figure $6(\mathrm{~b})$, a four-layer structure was used in the optical model (air/processed a-C:H/non processed a-C: 


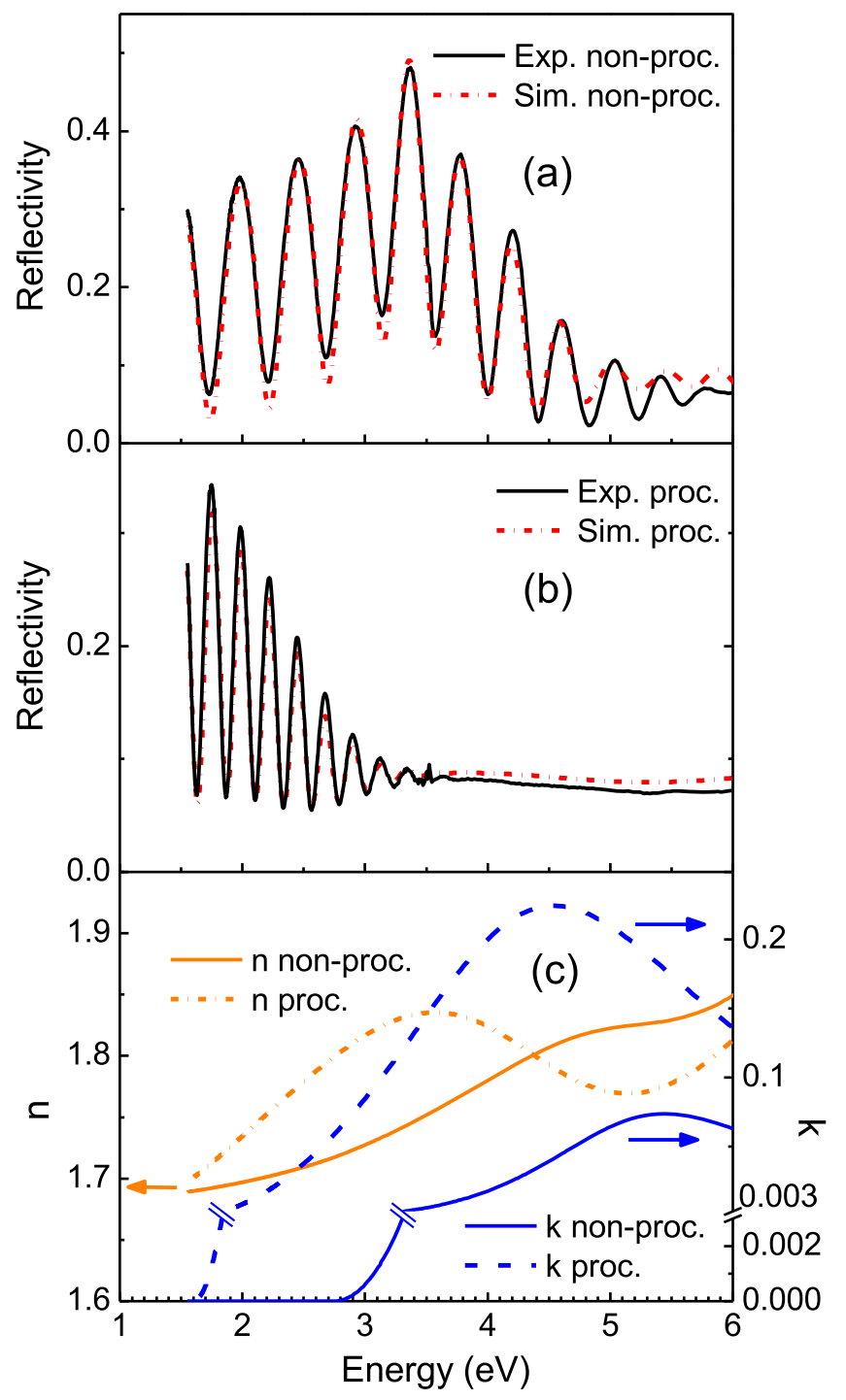

Figure 6. Reflectivity spectra and optical indices of a-C:H samples. Experimental reflectivity spectrum and model simulation (see text) of (a) a $750 \mathrm{~nm}$ thick non-irradiated a-C:H and (b) a $1540 \mathrm{~nm}$ thick irradiated a-C:H layer (fluence $\approx 4.3 \times 10^{17} \mathrm{e} \mathrm{cm}^{-2}$ ). An image of the processed sample can be found in figure 3. (c) Optical indices derived from the optical model for all the studied samples. The Tauc gap energies are $E_{\mathrm{g}}=2.7 \mathrm{eV}$ for unprocessed samples and $E_{\mathrm{g}}=1.6 \mathrm{eV}$ for processed samples.

$\mathrm{H} / \mathrm{Si}$ ). The optical indices of the processed material are described again with the mentioned Cauchy and TaucLorentz models. The absorption (given by the $k$ value) is much higher for the processed sample $(k=0.22$ at the maximum, $4.5 \mathrm{eV}$ as compared with the $k=0.074$ value for unprocessed material). The band gap energy of the processed sample, $E_{\mathrm{g}}=1.6 \mathrm{eV}$, corresponds to photons of $775 \mathrm{~nm}$. Most visible photons are thus absorbed by the sample as illustrated by the dark color observed for the irradiated area in figure 3. The optical indices in figure 6(c) give a good fit to the reflectivity spectra of the five a-C:H samples analyzed (not shown for brevity), both before and after electron processing. A decrease of the Tauc gap upon energetic processing was also observed in the work of Gadallah et al [37],

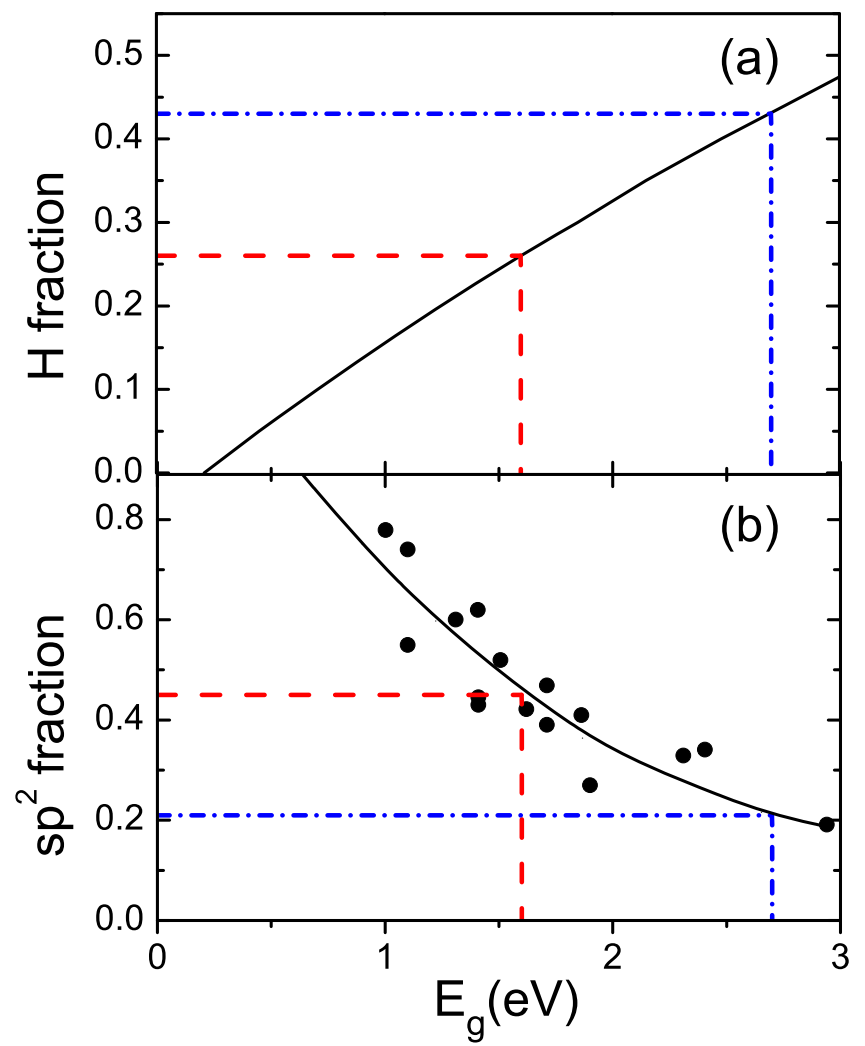

Figure 7. Correlations between the band gap energy (Tauc gap) and the a-C:H atomic composition. The blue dash-dot line corresponds to the unprocessed samples of this work, the red dash line to the processed samples. (a) Black solid line: $\mathrm{H}$ fraction,

$\mathrm{H} /\left(\mathrm{H}+\mathrm{Csp}^{3}+\mathrm{Csp}^{2}\right)$, versus band gap energy, adapted from [38]. (b) Black dots $\mathrm{sp}^{2}$ fraction, $\mathrm{Csp}^{2} /\left(\mathrm{Csp}^{2}+\mathrm{Csp}^{3}\right)$ versus band gap energy, adapted from [8]. The black solid line has been drawn through the experimental data.

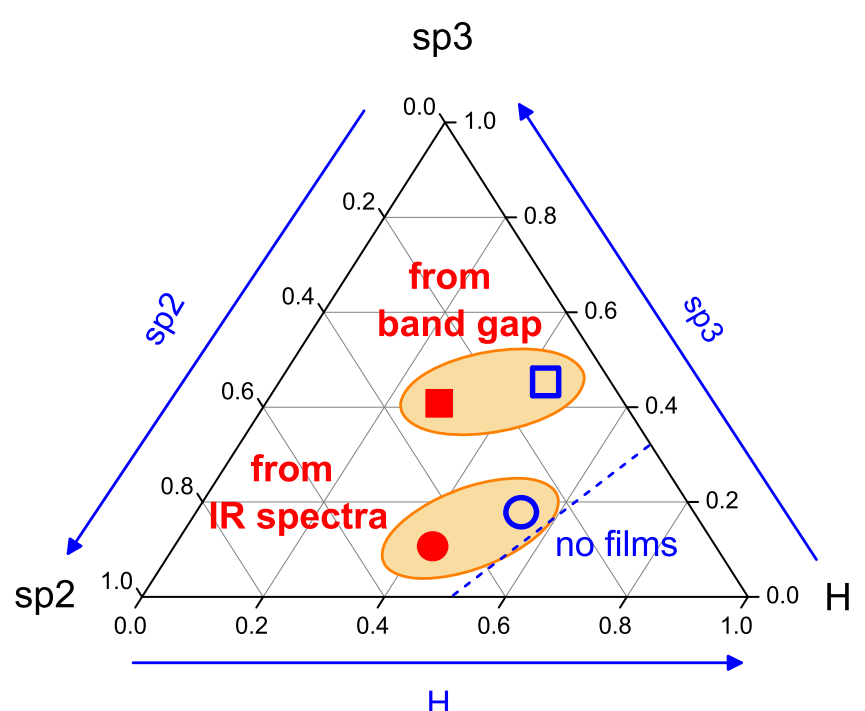

Figure 8. Ternary diagram for a-C:H with estimates of the atomic compositions of the a-C:H samples produced in this work. Open blue symbols, unprocessed deposits. Closed red symbols, processed samples (see tables 1 and 2). 
Table 2. Atomic composition of plasma generated a-C:H before and after energetic processing with $5 \mathrm{keV}$ electrons. The compositions were estimated from the band gap values derived from the modeling of reflectivity spectra (see text) and the correlations given by Robertson and co-workers [8, 38]. The values listed correspond to the average of measurements performed on five samples with different thickness (between 380 and $1540 \mathrm{~nm}$, respectively). The irradiation fluences varied between $\approx 2$ and $2.2 \times 10^{17} \mathrm{e} \mathrm{cm}^{-2}$ for samples with thickness $<1000 \mathrm{~nm}$ and $\approx 4.3-4.65 \times 10^{17} \mathrm{e} \mathrm{cm}^{-2}$ for thicker samples.

\begin{tabular}{lcc}
\hline & \multicolumn{2}{c}{ Composition from reflectivity measurements } \\
\cline { 2 - 3 } & $\begin{array}{c}\text { a-C:H as deposited by } \\
\text { PECVD }\end{array}$ & $\begin{array}{c}\text { a-C:H after electron } \\
\text { bombardment }\end{array}$ \\
\hline $\mathrm{C} \mathrm{sp}^{3}$ & 0.44 & 0.41 \\
$\mathrm{C} \mathrm{sp}$ & 0.11 & 0.33 \\
$\mathrm{H}$ & 0.45 & 0.26 \\
\hline
\end{tabular}

where a-C:H samples generated by laser ablation of graphite in a hydrogen atmosphere, were irradiated with UV photons.

The relationship between the composition, structure and general properties of hydrogenated amorphous carbon has been thoroughly studied by Robertson and co-workers (see for instance [5, 8, 35] and references therein). From these studies, useful approximate correlations between the Tauc band gap and the contents of hydrogen or the carbon hybridization were derived. In figure 7, we have represented two of these correlations. The black solid line of figure 7(a) represents the correlation of the Tauc gap as a function of $\mathrm{H}$ content reported by Casiraghi et al [38] from a very large set of measurements on a-C:H solids of very different compositions. Although a single curve is proposed to encompass all the data, it should be noted that the dispersion of data around this curve is large (see figure 7 of [38]). In figure 7(b), the points represent the experimental data gathered in [8] for typical a-C:H solids obtained by PECVD, and the line has just been drawn through them. Note that, as expected, the band gap drops with growing $\mathrm{sp}^{2}$ fraction and with decreasing $\mathrm{H}$ atom content, as the material becomes more graphitic. We have used these correlations to estimate the approximate atomic compositions of our samples.

The results are summarized in table 2. This analysis based on reflectivity measurements also shows that the atomic proportions change appreciably with electron irradiation. There is significant dehydrogenation and an increase in the proportion of $\mathrm{Csp}^{2}$ and, thus, a growth of aromatic structures in the solid.

Although both IR and reflectivity measurements reflect qualitatively the same trend toward dehydrogenation and graphitization of the a-C:H solids upon energetic electron processing, the actual atomic compositions derived by the two methods before and after processing are rather different. This is best seen in the ternary diagram represented in figure 8 . The two methods lead to hydrogen-rich ('polymer-like' [38]) a-C: $\mathrm{H}$ for the unprocessed samples, which tend to a-C:H films with intermediate $\mathrm{H}$ content after electron irradiation, but the proportion of $\mathrm{sp}^{3}$ bonding obtained from the reflectivity measurements and band gap correlations is much higher than

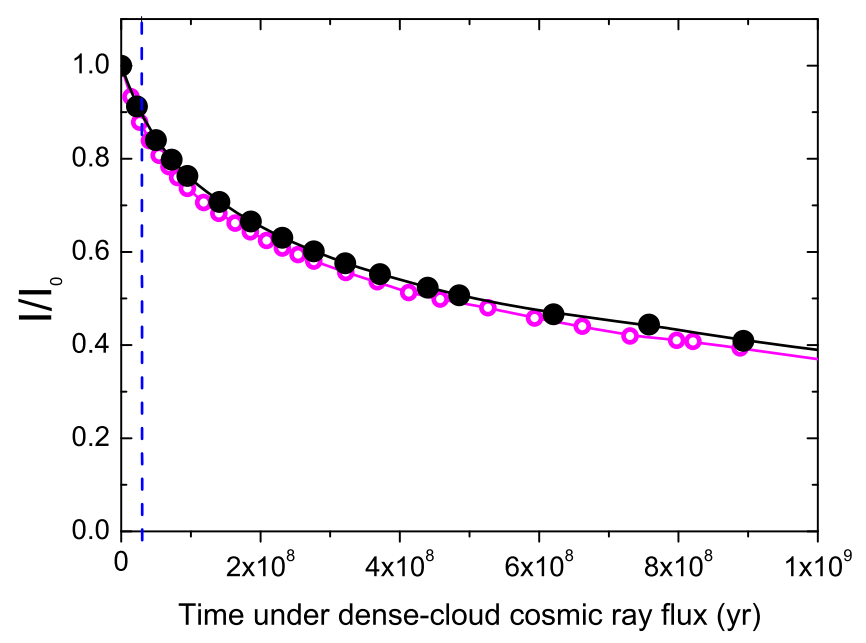

Figure 9. Decay of the $\mathrm{CH}$ stretching $(3.4 \mu \mathrm{m})$ band intensity under the effect of cosmic rays for typical dense cloud conditions (see text). Black dots and line: present results. Small pink circles and line: results from [26]. Blue dashed line: typical lifetime of a dense cloud.

that from the IR data. In a previous work [19], we concluded from a comparison between theoretical and measured IR spectra that the results of the IR estimates could be biased toward graphitic structures. The present discrepancy between estimates from IR and optical data points also in this direction. Composition estimates from table 2 are probably more reliable and in better agreement with the range of values reported in the literature $[7,8,32]$ for the type of a-C:H produced in our plasmas. However, one should also be cautious with the estimates from optical measurements because the atomic compositions derived with this method seem too 'diamond-like' and are not in good agreement with the theoretical results commented on in the discussion of figure 1 and, besides, the band gap correlations in figure 7 are only approximate. Although the two experimental procedures and the theoretical estimates agree on the high proportion of hydrogen $(45 \%-55 \%)$ present in the a-C:H samples used as IS carbonaceous dust analogs, more work is needed to clarify the predominant bonding in the carbon network.

\section{Astrophysical implications}

The energetic processing of a-C:H with high-energy electrons provides a means to estimate the effects of CRs on carbonaceous IS dust. Cosmic rays are mostly made of protons, but to a first approximation the effects of CRs on matter depend rather on the energy dose imparted than on the specific nature of the bombarding particles $[39,40]$. In a previous study, we irradiated a-C:H with $5 \mathrm{keV}$ electrons and concluded, in agreement with Goddard et al [22] who used multiple ions with different energies, that CRs cannot account for the disappearance of the $3.4 \mu \mathrm{m}$ band inside dense IS clouds. The present experiments corroborate our previous measurements (see figure 5) and therefore confirm the irrelevance of CRs for the destruction of the $3.4 \mu \mathrm{m}$ band carriers, which are mostly aliphatic $\mathrm{CH}_{n}$ groups. In the following, we review this 
conclusion using a different procedure for the estimation of characteristic times.

The amount of energy per unit time and unit mass deposited by CRs on carbonaceous grains inside dense clouds, $R_{\mathrm{CR}}$, can be expressed as:

$$
R_{\mathrm{CR}}=\Phi \times S
$$

where $\Phi$ is the CR flux and $S$ the stopping cross section of the material. We will make the usual assumption that the flux of multiple ions of variable energies in CRs can be approximated by an equivalent flux of $1 \mathrm{MeV}$ protons. Inside dense clouds, this flux is assumed to be $\Phi=1$ proton $\mathrm{cm}^{-2} \mathrm{~s}^{-1}[21,41]$. The stopping cross section of our a-C:H dust analogs $\left(\rho=1 \mathrm{~g} \mathrm{~cm}^{-3}, \quad \mathrm{C} / \mathrm{H}=1\right)$ for $1 \mathrm{MeV}$ protons is $S$ $(1 \mathrm{MeV})=262 \mathrm{MeV} \mathrm{cm}^{2} \mathrm{~g}^{-1}$, as calculated with the stopping and range of ions in matter code (SRIMS) [42]. This gives: $R_{\mathrm{CR}}=262 \mathrm{MeV} \mathrm{g}^{-1} \mathrm{~s}^{-1}=8.26 \times 10^{15} \mathrm{eV} \mathrm{g}^{-1} \mathrm{yr}^{-1}$ for $\mathrm{a}-\mathrm{C}$ : $\mathrm{H}$ gains in dense clouds. We can now use this value to scale the energy absorbed by the laboratory analogs under high-energy electron bombardment. At a given time, the energy per unit mass deposited by the electrons in the laboratory a-C:H layers, $\Delta E(t)$, can be expressed as:

$$
\Delta E(t)=F(t) \times S_{e},
$$

where $F(t)$ is the electron fluence at time $t$, and $S_{e}$ the stopping cross section of the a-C:H samples for $5 \mathrm{keV}$ electrons. Calculations using the CASINO program mentioned above yield [26]: $S_{e}=79 \mathrm{MeV} \mathrm{cm}^{2} \mathrm{~g}^{-1} \mathrm{~s}^{-1}$ for our dust analogs. We can now express the decay of the $3.4 \mu \mathrm{m}$ band of a-C:H dust, due to CR bombardment inside a dense cloud, as a function of time. The dense-cloud time corresponding to a given fluence is given by:

$$
t=\frac{S_{e}}{R_{\mathrm{CR}}} \times F .
$$

The result of the fluence to time transformation is represented in figure 9. With the CR energy dose expected for a dense cloud, the decay of the band to $50 \%$ of the initial intensity would take about $5 \times 10^{8}$ years. However, the lifetime of a typical dense cloud, before collapsing to a protostar is estimated to be $\approx 3 \times 10^{7}$ yr [22]. Over this period of time, the decrease in intensity is of the order of just 10\% (see dashed line in figure 9). The present results show once more that $\mathrm{CR}$ irradiation cannot justify the observed disappearance of the $\mathrm{CH}$ band in dense clouds.

\section{Summary and conclusions}

Films of a-C:H were produced by PECVD of mixtures of $\mathrm{CH}_{4}+\mathrm{He}$ in an inductively coupled $\mathrm{RF}$ discharge. The deposition conditions were selected to produce films whose IR spectra were in good agreement with astronomical observations of IS carbonaceous dust, in particular with the characteristic $3.4 \mu \mathrm{m}$ band attributed mostly to $\mathrm{CH}$ stretching vibrations of $\mathrm{CH}_{2}$ and $\mathrm{CH}_{3}$ aliphatic groups.

The films were characterized using SEM, IR spectroscopy and UV-visible reflectivity measurements. DFT calculations of structures and IR spectra were also performed. The results of the analyses showed that the deposited films were homogeneous, with flat surfaces and with a high proportion of hydrogen $(\approx 50 \%)$ in their composition. The $\mathrm{Csp}^{2} / \mathrm{Csp}^{3}$ ratio based on IR spectroscopy is 1.5 whereas that from reflectivity measurements is 0.25 . The discrepancy is large even considering the approximate nature of the estimates. The reasons for this discrepancy are not clear but probably lie in the assumptions, models and empirical correlations used for the interpretation of the measurements. More work is needed to clarify the carbon bonding structure in laboratory IS dust analogs.

Irradiation of the samples with $5 \mathrm{keV}$ electrons leads to the destruction of aliphatic $\mathrm{CH}_{n}$ groups (decay of the $3.4 \mu \mathrm{m}$ band) and the formation of aromatic structures (growth of bands at $6.2 \mu \mathrm{m}$ and $11-12 \mu \mathrm{m}$ ). The results are consistent with a dehydrogenation model which assumes that electron bombardment induces $\mathrm{CH}$ breaking and that most of the liberated $\mathrm{H}$ atoms form $\mathrm{H}_{2}$ molecules that diffuse out of the solid. Part of the $\mathrm{H}$ atoms however get trapped again at reactive sites and remain in the network. Some of these $\mathrm{H}$ atoms give rise to $\mathrm{CH}$ bonds at the edges of the newly formed aromatic structures as evidenced by the growth of a band at $11-12 \mu \mathrm{m}$ which corresponds to aromatic $\mathrm{CH}$ bending vibrations.

The decay of the $3.4 \mu \mathrm{m}$ band of a-C:H under highenergy electron bombardment is comparatively slow. Estimates based on the absorbed energy and on models of cosmic ray flux indicate that CRs cannot account for the observed disappearance of this band in dense IS clouds and corroborate thus the results from previous works $[22,26]$.

\section{Aknowledgements}

This work was funded by the Spanish MINECO under grants FIS2013-48087-C2-1P and FIS2016-77726-C3-1P. European Union funding under the project ERC-2013-Syg 610256 (NANOCOSMOS) is also acknowledged. The authors would like to thank R Serna for useful discussions and support with the reflectivity measurements and WVASE program. Our skillful technicians M A Moreno, A González and J Rodríguez are also gratefully acknowledged.

\section{ORCID iDs}

R Escribano (i) https://orcid.org/0000-0002-3048-9420

V J Herrero (iD https://orcid.org/0000-0002-7456-4832

\section{References}

[1] van Dishoeck E F 2014 Faraday Discuss. 168 9-47

[2] Jones A P, Fanciullo L, Köhler M, Verstraete L, Guillet V, Bocchio M and Ysard N 2013 Astron. Astrophys. 558 A62

[3] Duley W W and Williams D A 1983 Mon. Not. R. Astron. Soc. 20567 
[4] Jones A P, Duley W W and Williams D A 1990 Q. J. R. Astron. Soc. 31567

[5] Robertson J 1986 Adv. Phys. 33317

[6] Angus J C and Jansen F 1988 J. Vac. Sci. Technol. A 61778

[7] Jacob W 1998 Thin Solid Films 326 1-42

[8] Robertson J 2002 Mat. Sci. Eng. R 37129

[9] Dartois E, Muñoz-Caro G M, Deboffle D, Montagnac G and d’Hendecourt L 2005 Astron. Astrophys. 432895

[10] Dartois E, Geballe T R, Pino T, Cao A T, Jones A, Deboffle D, Guerrini G, Bréchingac P and d’Hendecourt L 2007 Astron. Astrophys. 463635

[11] Kwok S and Zhang Y 2013 Astrophys. J. 7715

[12] Chiar J E, Tielens A G G M, Adamson A J and Ricca A 2013 Astrophys. J. 77078

[13] Steglich M, Jäger C, Huisken F, Friedrich M, Plass W, Räder H J, Müllen K and Henning T 2013 Astrophys. J. Suppl. Ser. 20826

[14] Pendleton Y J and Allamandola L J 2002 Astrophys. J. Suppl. Ser. 13875

[15] Jäger C, Krasnokutsky S, Staicu A, Huisken F, Mutschke H, Henning T, Poppitz W and Voicu I 2006 Astrophys. J. Suppl. Ser. 166557

[16] Kovacevic E, Stefanovic I, Berndt J, Pendleton Y J and Winter J 2005 Astrophys. J. 623242

[17] Maté B, Tanarro I, Moreno M A, Jiménez-Redondo M, Escribano R and Herrero V J 2014 Faraday Discuss. 168267

[18] Jones A P, Fanciullo L, Köhler M, Verstraete L, Guillet V, Bocchio M and Ysard N 2013 Astron. Astrophys. 556 A62

[19] Molpeceres G, Timón V, Jiménez-Redondo M, Escribano R, Maté B, Tanarro I and Herrero V J 2017 Phys. Chem. Chem. Phys. 19 1352-60

[20] Mennella V et al 2001 Astron. Astrophys. 367355

[21] Mennella V, Baratta G A, Esposito A, Ferini G and Pendleton Y J 2003 Astrophys. J. 87727

[22] Godard M et al 2011 Astron. Astrophys. 529 A146

[23] Gadallah K A K, Mutschke H and Jäger C 2012 Astron. Astrophys. 544 A107
[24] Alata I, Cruz-Diaz G A, Muñoz Caro G M and Dartois E 2014 Astron. Astrophys. 569 A119

[25] Mennella V, Brucato J R, Colangeli L and Palumbo P 2002 Astrophys. J. 569531

[26] Maté B, Molpeceres G, Jiménez-Redondo M, Tanarro I and Herrero V J 2016 Astrophys. J. 83151

[27] Clark S J, Segall M D, Pickard C J, Hasnip P J, Probert M J, Refson K and Payne M C 2005 Z. Krist. 220567

[28] Refson K, Tulip P R and Clark S J 2006 Phys. Rev. B 73 155114

[29] Drouin D et al 2007 Scanning 2992

[30] Demers H, Poirer-Demers N, Couture A R, Joly D, Guilmain M, de Jonge N and Drouin D 2011 Scanning 33135

[31] Schwarz-Selinger T, Von Keudell A and Jacob W 1999 J. Appl. Phys. 863988

[32] Robertson J 1992 Surf. Coat. Technol. 50185

[33] Hudggins D M and Allamandola M J 1999 Astrophys. J. 516141

[34] Adel M E, Amir O, Kalish R and Feldman L C 1989 J. Appl. Phys. 663248

[35] Marée C H M, Vredenberg A M and Habraken F H P M 1996 Mater. Chem. Phys. 46198

[36] Tompkins H and Eugene A I 2005 Handbook of Elipsometry (New York: William Andrews)

[37] Gadallah K A K, Mutschke H and Jäger C 2011 Astron. Astrophys. 528 A56

[38] Casiraghi C, Ferrari A C and Robertson J 2005 Phys. Rev. B 72 085401

[39] Kaiser R I, Stockton A M, Kim Y S, Jensen E C and Mathies A R A 2013 Astrophys. J. 756111

[40] Mason N J, Nailr B, Jheeta S and Szimańska E 2014 Faraday Discuss. 168235

[41] Moore M H, Hudson R L and Gerakines P A 2001 Spechtrochim. Acta A 57843

[42] Ziegler F J, Ziegler M D and Biersack J P 2010 Nucl. Instrum. Methods Phys. Res. B 2681818 